\title{
An Investigations of Sound Absorbance Properties of Weft Knitted Spacer Fabrics
}

\author{
Erhan Sancak \\ Marmara University Technology Faculty, Department of Textile Engineering, Istanbul, Turkey
}

(Received 2 November 2013; revised 22 March 2014; accepted 30 April 2014)

\begin{abstract}
Nonwoven fabrics have been used for many years in different technical applications; they have particularly been used as isolation materials in vehicles in order to reduce the noise heard within them, and they. They have achieved great popularity due to low production cost and good absorbance. However, the fabrics produced by making use of the nonwoven technique have some disadvantages including low resistance, low abrasion strength, poor aesthetic appearance and thickness. In order to eliminate these disadvantages, recent studies have reported that knitted fabrics could be an alternative to nonwoven fabrics. Various studies have focused on the impact on sound absorbance that the thickness and surface structure of knitted fabrics have. In this study, a number of knitted spacer fabrics, which had five different connection angles, were manufactured by using a plain knitting machine. The sound transmission loss levels of the developed fabrics were tested and analysed by Brüel and Kjaer tube instruments. At the end of the examinations, the sound absorbance behaviors at different frequencies were demonstrated in graphics based on the type of knitting. It was determined in the study that three factors have a major impact on the sound absorbance behaviour; thickness of fabric, micro porosity between fabric surfaces and yarn linear density in the interconnection of the fabrics.
\end{abstract}

\section{INTRODUCTION}

In humans, the audible range of sound frequencies is usually $20 \mathrm{~Hz}$ to $20.000 \mathrm{~Hz}$. For a sound to be heard, the sound intensity should reach a certain level. Human sounds usually cover the frequencies of 250-500-1000-2000 Hz. If these audio waves appear in a random spectrum or, in other words, if they are undesired sounds, they are called "noise".

In parallel to technological advancements, the development of metropolitan regions, and the lightening of structures, environmental impacts that affect humans are also on the rise. One of these notable impacts is the sound pollution, or environmental noise, which is irritating, undesirable, and uncomfortable. In addition to the discomfort that it causes, it also has negative impacts on the psychology, physiology, and performance of humans. Besides, noise can result in behavioral disorders, decline in working performance, hearing loss, tinnitus and psychological diseases.

In the urbanized and mobilized life style of today's world, the time spent within cars has increased. Thus, sound insulation has gained an increasing importance in the automobile industry for both the comfort of driver and the health of passenger. With the increase in the power of engines used in the automobile industry, low-frequency sound within the car has appeared to be a problematic area that needs to be resolved. Although this frequency range is generally below $4000 \mathrm{~Hz}$, sounds in the range of $100-1000 \mathrm{~Hz}$ cause passengers to feel tired. ${ }^{1}$

If a sound encounters an obstacle while propagating in a medium, it behaves in three different manners as in the case of other physical phenomena. A certain part of the sound reflects from the obstacle, and the obstacle absorbs a certain amount of it while the rest passes through the obstacle. Therefore, each material has a specific absorbance coefficient. As textile materials are porous structures, they allow sound absorbance. Thus, they are used in a wide range of applications including acoustic panels used in workplaces, insulation materials for automobiles and furnishing fabrics in the concert halls.,

In industrial applications, fibreglass, foam, mineral fibres and their composite materials are used for sound insulation.

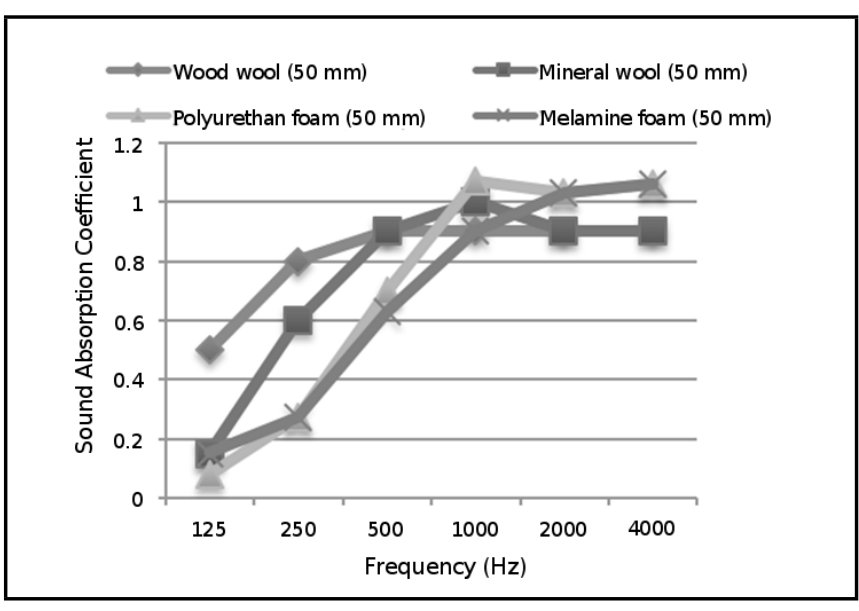

Figure 1. Sound Absorbance Behaviours of Certain Materials. ${ }^{2}$

Even though these materials have good heat and sound insulation properties, they have negative impacts in terms of environmental pollution and human health.

In the transportation sector, manufacturers have used three main methods for sound control and sound reduction within the car. These are the reduction of sound and vibration sources, establishing anti-sound barriers between the passenger and the sound source, and using absorbent materials for sound propagation inside and outside the car. ${ }^{2}$

Nonwoven textile fabrics are commonly used in automobiles for sound insulation, as they are cost effective. However, it is less likely to manufacture nonwoven textile products as preformed or in the desired form. On the other hand, knitted fabrics have an aesthetic appearance, and they provide the opportunity to be manufactured in the desired form. The sound insulation capacity of single-plate knitted fabrics is poor. ${ }^{1}$ Thus, it is more appropriate to use double plate special patterned fabrics in the knitted fabrics for sound insulation.

Two different conclusions were drawn at the end of the measurements of the sound absorbance coefficients of the different spacer fabrics, which were manufactured out of 972 dtex yarn with double polyamide coating in the exterior face and elas- 